Portland State University

PDXScholar

Fall 12-29-2015

\title{
A Method for Achieving Analytic Formulas for Three Body Integrals Consisting of Powers and Exponentials in All Three Interparticle Hyllerass Coordinates
}

Chris M. Keating

Portland State University

Follow this and additional works at: https://pdxscholar.library.pdx.edu/open_access_etds

Part of the Quantum Physics Commons

Let us know how access to this document benefits you.

\section{Recommended Citation}

Keating, Chris M., "A Method for Achieving Analytic Formulas for Three Body Integrals Consisting of Powers and Exponentials in All Three Interparticle Hyllerass Coordinates" (2015). Dissertations and Theses. Paper 2638.

https://doi.org/10.15760/etd.2634

This Thesis is brought to you for free and open access. It has been accepted for inclusion in Dissertations and Theses by an authorized administrator of PDXScholar. Please contact us if we can make this document more accessible: pdxscholar@pdx.edu. 
A Method for Achieving Analytic Formulas for Three Body Integrals Consisting of Powers and Exponentials in All Three Interparticle Hylleraas Coordinates

by

Chris M Keating

A thesis submitted in partial fulfillment of the requirements for the degree of

\author{
Master of Science \\ in \\ Physics \\ Thesis Committee: \\ Jack C Straton \\ Peter Leung \\ Andres La Rosa
}

Portland State University

2015 
(C)2015 Chris M Keating 


\begin{abstract}
After an introduction to the variational principle of three body systems via the Helium atom, we present general analytical formulas for the radial parts of integrals that occur when three body systems are described using wave functions that consist of powers and exponentials in all three interparticle Hylleraas coordinates [1]. This work is an extension of integrals given by Harris, Frolov and Smith, Jr. [2]. Specifically included are radial integrals encountered in calculations involving the dipole moment matrix element in Hylleraas coordinates that contain a function $f\left(k r_{1}\right)$ (such as a spherical Bessel function) in addition to a plane wave, a hydrogenic orbital and exponentials in all three interparticle coordinates.
\end{abstract}




\section{Acknowledgements}

Keating wishes to thank Anand K Bhatia for his helpful comments, as well as Jack C Straton for his advice and continual guidance. 


\section{Table of Contents}

Abstract................................................... page $i$

Acknowledgements.................................... page ii

Introduction.......................................... page 1

Section 1

The Ground State of Helium..................... page 2

Using the Variational Principle.................. page 5

Hylleraas Coordinates........................... page 7

Section 2

Photodetachment................................ page 13

Definitions...................................... page 14

Integrals $\Lambda_{l m n}(\alpha, \beta, \gamma ; k) \ldots \ldots \ldots \ldots \ldots \ldots \ldots \ldots \ldots$ page 15

Integrals $\Lambda_{l m n}^{11}(\alpha, \beta, \gamma ; k) \ldots \ldots \ldots \ldots \ldots \ldots \ldots \ldots . \ldots$ page 16

Integrals $\Lambda_{l m n}^{12}(\alpha, \beta, \gamma ; k) \ldots \ldots \ldots \ldots \ldots \ldots \ldots \ldots \ldots$ page 18

Integrals $J_{L M N}(a, b, c ; k) \ldots \ldots \ldots \ldots \ldots \ldots \ldots \ldots$ page 21

General Approach.................................... page 22

Conclusion......................................... page 25

References........................................... page 26

Appendix................................................ page 30

Derivation of the Hamiltonian in Hylleraas Coordinates 


\section{Introduction}

This paper consists of two main sections. The first section is an introduction to the variational principle for three body systems, which is an approximation technique that provides accurate ground state energies and wavefunctions. Once the accurate ground state wavefunctions are found by the variational principle, the second section is a novel way of obtaining closed form analytical formulas for the matrix elements of a dipole transition, $\mu_{z}=\int \psi_{d}^{*}\left(z_{1}+z_{2}\right) \psi_{c} d \tau$. This leads directly to the atomic absorption coefficient, $\sigma=\left(6.812 \times 10^{-20} \mathrm{~cm}^{2}\right) k\left(k^{2}+2 I\right)\left|\mu_{z}\right|^{2}$, an important quantity of interest for photodetachment. 


\section{Section 1}

\section{The Ground State of Helium}

The Helium atom consists of two electrons in orbit around a nucleus that contains two protons. The non-relativistic Hamiltonian, neglecting the motion of the nucleus and given in atomic units, is

$$
H=-\frac{1}{2}\left(\nabla_{r_{1}}^{2}+\nabla_{r_{2}}^{2}\right)-\frac{2}{r_{1}}-\frac{2}{r_{2}}+\frac{1}{\left|\mathbf{r}_{1}-\mathbf{r}_{2}\right|}
$$

where $r_{1}$ and $r_{2}$ denote the electron distances from the nucleus [3].

In this study, we would like to determine the ground state energy (the amount of energy it would take to strip off the two electrons) and ground state wavefunction for this system. The ground state energy has been measured with great precision in the laboratory. The experimental value for the ground state energy of helium is $E=$ $-79.005151042 \mathrm{eV}$ [4]. This is the number we would like to reproduce theoretically. In many other three-body problems with non-Coulombic potentials there have been found exact solutions. For an example of "heliumlike" potentials that have exact solutions see Crandall, Whitnell and Betteha [5]. Straton has demonstarated the usefulness of integral transforms for determining analytically reduced forms for a general class of integrals containing multicenter products of 1 s hydrogenic orbitals, Yukawa or Coulomb potentials, and plane waves [6][7][8][9]. Yet, it is an unfortunate

fact that no exactly solvable solutions (in terms of algebraic expressions) exist for the helium atom.

The feature that makes solving this system difficult is the electron/electron repulsion term. Therefore, we will start by ignoring the electron/electron repulsion term. Thus, the approximated Hamiltonian is, 


$$
H=-\frac{1}{2}\left(\nabla_{r_{1}}^{2}+\nabla_{r_{2}}^{2}\right)-\frac{2}{r_{1}}-\frac{2}{r_{2}}
$$

With the electron/electron repulsion term ignored, the Hamiltonian is just two independent hydrogen Hamiltonians with twice the Coulomb potential. Let us assume two 1s hydrogen electrons have the radial wavefunction:

$$
\psi_{100}\left(\mathbf{r}_{1}\right) \psi_{100}\left(\mathbf{r}_{2}\right)=\psi_{H e}\left(\mathbf{r}_{1}, \mathbf{r}_{2}\right)=A e^{-\lambda\left(r_{1}+r_{2}\right)}
$$

where $\lambda$ equals $Z / a_{0}$. We write the 1 s hydrogen electron ground state in the form,

$$
\psi_{100}(\mathbf{r})=\frac{\lambda^{3 / 2}}{\sqrt{\pi}} e^{-\lambda r}
$$

Thus the normalization constant for the total system is,

$$
A=\frac{\lambda^{3}}{\pi}
$$

Using the eigensystem equation,

$$
H \psi=E \psi,
$$

the ground state wavefunction is spherically symmetric. Thus, the angular part is constant and we find

$$
\begin{gathered}
H \psi=-\frac{1}{2}\left(\frac{1}{r_{1}^{2}} \frac{\partial}{\partial r_{1}}\right. \\
\left.\left(r_{1}^{2} \frac{\partial}{\partial r_{1}}\right) A e^{-\lambda\left(r_{1}+r_{2}\right)}+\frac{1}{r_{2}^{2}} \frac{\partial}{\partial r_{2}}\left(r_{2}^{2} \frac{\partial}{\partial r_{2}}\right) A e^{-\lambda\left(r_{1}+r_{2}\right)}\right) \\
-\frac{2}{r_{1}} A e^{-\lambda\left(r_{1}+r_{2}\right)}-\frac{2}{r_{2}} A e^{-\lambda\left(r_{1}+r_{2}\right)}
\end{gathered}
$$

or

$$
H \psi=\left[-\frac{1}{2}\left(\lambda^{2}-\frac{2 \lambda}{r_{1}}+\lambda^{2}-\frac{2 \lambda}{r_{2}}\right)-\frac{2}{r_{1}}-\frac{2}{r_{2}}\right] A e^{-\lambda\left(r_{1}+r_{2}\right)}
$$


The equality must hold for any $r_{1}$ and $r_{2}$,

$$
\begin{aligned}
-\frac{1}{2} \lambda^{2}+\frac{\lambda}{r_{1}}-\frac{1}{2} \lambda^{2}+\frac{\lambda}{r_{2}}-\frac{2}{r_{1}}-\frac{2}{r_{2}} & =E \\
-\lambda^{2}+\frac{\lambda-2}{r_{1}}+\frac{\lambda-2}{r_{2}} & =E
\end{aligned}
$$

Therefore, $\lambda=2$, and $E=-4$ a.u. Using 1 a.u. $=27.211 \mathrm{eV}$, we find $E=-108.8 \mathrm{eV}$.

However, the above solution ignored the electron/electron repulsion. The electron/electron repulsion term is [10]

$$
V_{12}=\frac{1}{\left|\mathbf{r}_{1}-\mathbf{r}_{2}\right|}=\frac{4 \pi}{2 l+1} \sum_{l=0}^{\infty} \sum_{m=-l}^{l} \frac{r_{<}^{l}}{r_{>}^{l+1}} Y_{l m}\left(\theta_{1}, \phi_{1}\right) Y_{l m}^{*}\left(\theta_{2}, \phi_{2}\right)
$$

with expectation value

$$
<V_{12}>=A^{2} \iint \frac{e^{-2 \lambda\left(r_{1}+r_{2}\right)}}{\left|\mathbf{r}_{1}-\mathbf{r}_{2}\right|} d^{3} \mathbf{r}_{1} d^{3} \mathbf{r}_{2}
$$

or

$$
\begin{aligned}
<V_{12}>=A^{2} \sum_{l=0}^{\infty} \sum_{m=-l}^{l} \frac{4 \pi}{2 l+1} \iint e^{-2 \lambda\left(r_{1}+r_{2}\right)} \frac{r_{<}^{l}}{r_{>}^{l+1}} r_{1}^{2} r_{2}^{2} d r_{1} d r_{2} \\
\times \iint Y_{l m}\left(\theta_{1}, \phi_{1}\right) \sin \theta_{1} d \theta_{1} d \phi_{1} \\
\times \iint Y_{l m}^{*}\left(\theta_{2}, \phi_{2}\right) \sin \theta_{2} d \theta_{2} d \phi_{2} .
\end{aligned}
$$

Looking at the first spherical part and knowing that $Y_{00}=Y_{00}^{*}=\frac{1}{\sqrt{4 \pi}}$, we have

$$
\iint Y_{l m}\left(\theta_{1}, \phi_{1}\right) \sin \theta_{1} d \theta_{1} d \phi_{1}=\sqrt{4 \pi} \iint Y_{l m}\left(\theta_{1}, \phi_{1}\right) Y_{00}^{*}\left(\theta_{1}, \phi_{1}\right) \sin \theta_{1} d \theta_{1} d \phi_{1}=\sqrt{4 \pi} \delta_{0 l} \delta_{0 m}
$$

Therefore Eq. (12) simplifies to 


$$
\begin{gathered}
<V_{12}>=A^{2} \sum_{l=0}^{\infty} \sum_{m=-l}^{l} \frac{4 \pi}{2 l+1} \iint e^{-2 \lambda\left(r_{1}+r_{2}\right)} \frac{r_{<}^{l}}{r_{>}^{l+1}} r_{1}^{2} r_{2}^{2} d r_{1} d r_{2} \sqrt{4 \pi} \delta_{0 l} \delta_{0 m} \sqrt{4 \pi} \delta_{0 l} \delta_{0 m} \\
=16 \pi^{2} A^{2} \iint e^{-2 \lambda\left(r_{1}+r_{2}\right)} \frac{1}{r_{>}} r_{1}^{2} r_{2}^{2} d r_{1} d r_{2} .
\end{gathered}
$$

In order to integrate this expression, we pick the limits of integration carefully,

$$
<V_{12}>=16 \pi^{2} A^{2} \int e^{-2 \lambda r_{2}}\left[\int_{0}^{r_{2}} e^{-2 \lambda r_{1}} r_{1}^{2} r_{2} d r_{1}+\int_{r_{2}}^{\infty} e^{-2 \lambda r_{1}} r_{1} r_{2}^{2} d r_{1}\right] d r_{2},
$$

which can also be shown to be

$$
\begin{gathered}
<V_{12}>=16 \pi^{2} A^{2} \int e^{-2 \lambda r_{2}}\left[\frac{r_{2}-e^{-2 \lambda r_{2}} r_{2}-\lambda e^{-2 \lambda r_{2} r_{2}^{2}}}{4 \lambda^{3}}\right] d r_{2} \\
=16 \pi^{2} A^{2} \frac{5}{128 \lambda^{5}} \\
=16 \pi^{2}\left(\frac{\lambda^{3}}{\pi}\right)^{2} \frac{5}{128 \lambda^{5}} \\
=\frac{5}{8} \lambda \\
=\frac{5}{4}
\end{gathered}
$$

in atomic units. Thus, $E=-4$ a.u. $+\frac{5}{4}$ a.u. $=-\frac{11}{4}$ a.u. or $E=-74.8 \mathrm{eV}$. This is an improvement on the first approximation, which ignores the electron/electron interaction.

\section{Using the Variational Principle}

In most cases, it is best to use the variational technique to improve the wavefunction. Let $\lambda$ be our variational parameter that will need to be minimized at the end of our calculation. Starting with the trial wavefuction,

$$
\psi_{H e}\left(\mathbf{r}_{1}, \mathbf{r}_{2}\right)=\frac{\lambda^{3}}{\pi} e^{-\lambda\left(r_{1}+r_{2}\right)}
$$


The expectation value is

$$
\begin{aligned}
& <H>=\iint\left[-\lambda^{2}+\frac{\lambda-2}{r_{1}}+\frac{\lambda-2}{r_{2}}\right] \frac{\lambda^{6}}{\pi^{2}} e^{-2 \lambda\left(r_{1}+r_{2}\right)} d^{3} \mathbf{r}_{1} d^{3} \mathbf{r}_{2}+\frac{5}{8} \lambda \\
& =\quad-\frac{\lambda^{8}}{\pi^{2}} \iint e^{-2 \lambda\left(r_{1}+r_{2}\right)} d^{3} \mathbf{r}_{1} d^{3} \mathbf{r}_{2} \\
& +(\lambda-2) \frac{\lambda^{6}}{\pi^{2}} \iint \frac{1}{r_{1}} e^{-2 \lambda\left(r_{1}+r_{2}\right)} d^{3} \mathbf{r}_{1} d^{3} \mathbf{r}_{2} \\
& +(\lambda-2) \frac{\lambda^{6}}{\pi^{2}} \iint \frac{1}{r_{2}} e^{-2 \lambda\left(r_{1}+r_{2}\right)} d^{3} \mathbf{r}_{1} d^{3} \mathbf{r}_{2}+\frac{5}{8} \lambda
\end{aligned}
$$

Let us do this piece by piece. The first integral is

$$
\begin{gathered}
\iint e^{-2 \lambda\left(r_{1}+r_{2}\right)} d^{3} \mathbf{r}_{1} d^{3} \mathbf{r}_{2} \\
=16 \pi^{2} \int e^{-2 \lambda r_{1}} r_{1}^{2} d r_{1} \int e^{-2 \lambda r_{2}} r_{2}^{2} d r_{2} \\
=16 \pi^{2} \frac{1}{4 \lambda^{3}} \frac{1}{4 \lambda^{3}} \\
=\frac{\pi^{2}}{\lambda^{6}} .
\end{gathered}
$$

Now, the second integral is

$$
\begin{gathered}
\iint \frac{1}{r_{1}} e^{-2 \lambda\left(r_{1}+r_{2}\right)} d^{3} \mathbf{r}_{1} d^{3} \mathbf{r}_{2} \\
=16 \pi^{2} \int e^{-2 \lambda r_{1}} r_{1} d r_{1} \int e^{-2 \lambda r_{2}} r_{2}^{2} d r_{2} \\
=16 \pi^{2} \frac{1}{4 \lambda^{2}} \frac{1}{4 \lambda^{3}} \\
=\frac{\pi^{2}}{\lambda^{5}} .
\end{gathered}
$$

Therefore,

$$
\begin{gathered}
<H>=-\frac{\lambda^{8}}{\pi^{2}} \frac{\pi^{2}}{\lambda^{6}}+(\lambda-2) \frac{\lambda^{6}}{\pi^{2}} \frac{\pi^{2}}{\lambda^{5}}+(\lambda-2) \frac{\lambda^{6}}{\pi^{2}} \frac{\pi^{2}}{\lambda^{5}}+\frac{5}{8} \lambda \\
=-\lambda^{2}+(\lambda-2) \lambda+(\lambda-2) \lambda+\frac{5}{8} \lambda \\
=\lambda^{2}-\frac{27}{8} \lambda .
\end{gathered}
$$

We find the minimum of this function to be:

$$
\frac{d}{d \lambda}<H>=2 \lambda-\frac{27}{8}=0
$$

or 


$$
\lambda=\frac{27}{16}=1.6875
$$

Thus, the ground state energy is

$$
E=-\frac{729}{256} \text { a.u. }=-2.848 \text { a.u. }=-77.5 \mathrm{eV}
$$

which is within $2 \%$ of the experimental value.

\section{Hylleraas coordinates}

The most common coordinates in which to compute the variational integrals are the Hylleraas coordinates [1]. In this coordinate system, one uses wavefunctions of the type

$$
\psi\left(r_{1}, r_{2}, r_{12}\right)=\frac{1}{\sqrt{2}}\left(1-\hat{P}_{12}\right) e^{-\alpha r_{1}-\beta r_{2}-\gamma r_{12}} \sum_{l, m, n} c_{l m n} s^{l} t^{2 m} u^{n}
$$

where $s=r_{1}+r_{2}, t=r_{1}-r_{2}, u=r_{12} \equiv\left|\mathbf{r}_{1}-\mathbf{r}_{2}\right|$ and $\hat{P}_{12}$ is the permutation operator for two identical electrons. The Hylleraas approach explicitly accounts for the interactional motions of the two electrons through the variable $u=r_{12}$. In fact the Hylleraas coordinate system is not a formal solution for the helium atom [11] [12], yet it has had great success in yielding accurate values for the ground state energies of three body systems. For example, a basis set of six wavefunctions could look like [13], 


$$
\begin{aligned}
& \psi_{1}=e^{-\alpha r_{1}-\beta r_{2}-\gamma r_{12}} \\
& \psi_{2}=e^{-\alpha r_{1}-\beta r_{2}-\gamma r_{12} u} \\
& \psi_{3}=e^{-\alpha r_{1}-\beta r_{2}-\gamma r_{12} t^{2}} \\
& \psi_{4}=e^{-\alpha r_{1}-\beta r_{2}-\gamma r_{12} s} \\
& \psi_{5}=e^{-\alpha r_{1}-\beta r_{2}-\gamma r_{12}} s^{2} \\
& \psi_{6}=e^{-\alpha r_{1}-\beta r_{2}-\gamma r_{12}} u^{2},
\end{aligned}
$$

where

$$
\psi=\sum_{i} c_{i} \psi_{i}
$$

From this, one can obtain $E=-2.90324$ a.u., which differs from the "exact" value by only 0.00048 a.u. In fact, the ground state of Helium is one of the most accurate theoretical numbers that has been calculated by quantum mechanical approximation methods. In particular, K. Frankowski and C.L. Pekeris obtained in 1966 the value $E=-2.9037243770326$ a.u. [14]. It should be noted that in this discussion we have ignored the the mass polarisation term $\left(-\frac{1}{M} \nabla_{r_{1}} \cdot \nabla_{r_{2}}\right)$ and relativistic correction term $\left(-\frac{\hat{p}^{4}}{8 c^{2}}\right)$ that are given in Pekeris' earlier work [15][16].

The usual volume element $d^{3} r_{1} d^{3} r_{2}=r_{1}^{2} r_{2}^{2} \sin \theta_{1} \sin \theta_{1} d r_{1} d r_{2} d \theta_{1} d \theta_{2} d \phi_{1} d \phi_{2}$, can be modified by referring to Euler angles. The Euler angles are defined as:

$$
\begin{gathered}
\theta_{1}=\Theta ; \phi_{1}=\Phi \\
\cos \theta_{2}=\cos \Theta \cos \Theta_{2}+\sin \Theta \sin \Theta_{2} \cos \Psi \\
\sin \theta_{2} \sin \left(\phi_{2}-\Phi\right)=\sin \Theta_{2} \sin \Psi \\
\cos \theta_{n}=\cos \Theta \cos \Theta_{n}+\sin \Theta \sin \Theta_{n} \cos \left(\Psi+\phi_{n}\right) \\
\sin \theta_{n} \sin \left(\phi_{n}-\Phi\right)=\sin \Theta \sin \left(\Psi+\phi_{n}\right) ; n>2,
\end{gathered}
$$

where $n$ allows one to generalize to more particles if needed. The motivation for using the Euler angles $\Theta, \Phi, \Psi$, is the important fact that the angles can be separated from 
one another and can be solved for more readily [17]. Using these angles transforms the volume element into

$$
d V=d^{3} r_{1} d^{3} r_{2}=r_{1}^{2} r_{2}^{2} d r_{1} d r_{2} \sin \theta_{1} \sin \theta_{12} d \theta_{1} d \theta_{12} d \phi_{1} d \Psi
$$

Since the functions involved depend only on $r_{1}, r_{2}$ and $r_{12}$, the angles $\phi_{1}, \theta_{1}$ and $\psi$ can be integrated, producing a factor of $8 \pi^{2}$, leaving

$$
d V=8 \pi^{2} r_{1}^{2} r_{2}^{2} d r_{1} d r_{2} \sin \theta_{12} d \theta_{12}
$$

Using, $r_{12}^{2}=r_{1}^{2}+r_{2}^{2}-2 r_{1} r_{2} \cos \theta_{12}$, we have that $r_{1} r_{2} \sin \theta_{12} d \theta_{12}=r_{12} d r_{12}$; consequently,

$$
d V=8 \pi^{2} r_{1} r_{2} r_{12} d r_{1} d r_{2} d r_{12}
$$

Note that the volume element is only a function of $r_{1}, r_{2}, r_{12}$. This allows integration over three coordinates rather than the usual six.

Let $P$ be the probability density such that,

$$
P=8 \pi^{2} \int_{0}^{\infty} \int_{0}^{\infty} \int_{\left|r_{1}-r_{2}\right|}^{r_{1}+r_{2}} \psi^{*} \psi r_{1} r_{2} r_{12} d r_{1} d r_{2} d r_{12}
$$

Note, the absolute values can be handled by

$$
\left|r_{1}-r_{2}\right|=\left\{\begin{array}{cc}
r_{1}-r_{2} & r_{1} \geq r_{2} \\
-\left(r_{1}-r_{2}\right) & r_{1}<r_{2}
\end{array}\right.
$$

Thus,

$$
P=8 \pi^{2} \int_{0}^{\infty}\left[\int_{0}^{r_{2}} \int_{-\left(r_{1}-r_{2}\right)}^{r_{1}+r_{2}} \psi^{*} \psi r_{1} r_{2} r_{12} d r_{12} d r_{1}+\int_{r_{2}}^{\infty} \int_{r_{1}-r_{2}}^{r_{1}+r_{2}} \psi^{*} \psi r_{1} r_{2} r_{12} d r_{12} d r_{1}\right] d r_{2}
$$


The Hamiltonian in the basis $\left(r_{1}, r_{2}, r_{12}\right)$ is

$$
\begin{gathered}
H=-\frac{1}{2} \frac{\partial^{2}}{\partial r_{1}^{2}}-\frac{1}{2} \frac{\partial^{2}}{\partial r_{2}^{2}}-\frac{\partial^{2}}{\partial r_{12}^{2}}-\frac{1}{r_{1}} \frac{\partial}{\partial r_{1}}-\frac{1}{r_{2}} \frac{\partial}{\partial r_{2}}-\frac{2}{r_{12}} \frac{\partial}{\partial r_{12}} \\
-\frac{r_{1}^{2}-r_{2}^{2}+r_{12}^{2}}{2 r_{1} r_{12}} \frac{\partial^{2}}{\partial r_{1} \partial r_{12}}-\frac{r_{2}^{2}-r_{1}^{2}+r_{12}^{2}}{2 r_{2} r_{12}} \frac{\partial^{2}}{\partial r_{2} \partial r_{12}} \\
-\left(\frac{Z}{r_{1}}+\frac{Z}{r_{2}}-\frac{1}{r_{12}}\right) .
\end{gathered}
$$

(See Appendix for derivation.)

The overlap integral matrix elements is defined as $\Delta_{i j}=\left\langle\psi_{i} \mid \psi_{j}\right\rangle$ or

$$
\Delta_{i j}=\left[\begin{array}{cccc}
\left\langle\psi_{1} \mid \psi_{1}\right\rangle & \left\langle\psi_{1} \mid \psi_{2}\right\rangle & \left\langle\psi_{1} \mid \psi_{1}\right\rangle & \ldots \\
\left\langle\psi_{2} \mid \psi_{1}\right\rangle & \left\langle\psi_{2} \mid \psi_{2}\right\rangle & \left\langle\psi_{2} \mid \psi_{3}\right\rangle & \ldots \\
\left\langle\psi_{3} \mid \psi_{1}\right\rangle & \left\langle\psi_{3} \mid \psi_{2}\right\rangle & \left\langle\psi_{3} \mid \psi_{3}\right\rangle & \ldots \\
\vdots & \vdots & \vdots & \ddots
\end{array}\right] .
$$

This is sometimes referred to as the metric because it shares many similar properties to those of $g_{u v}$, the gravitational tensor. Projecting the overlap integral onto the coordinate basis,

$$
\begin{aligned}
\left\langle\psi_{i} \mid \psi_{j}\right\rangle & =\left\langle\psi_{i}\left|\int\right| r\right\rangle\left\langle r|d V| \psi_{j}\right\rangle \\
& =\int\left\langle\psi_{i} \mid r\right\rangle\left\langle r \mid \psi_{j}\right\rangle d V \\
& =\int \psi_{i}^{*} \psi_{j} d V
\end{aligned}
$$

it can be seen that

$$
\left\langle\psi_{i} \mid \psi_{j}\right\rangle=8 \pi^{2} \int_{0}^{\infty} \int_{0}^{\infty} \int_{\left|r_{1}-r_{2}\right|}^{r_{1}+r_{2}} \psi_{i}^{*} \psi_{j} r_{1} r_{2} r_{12} d r_{1} d r_{2} d r_{12} .
$$

Likewise, for the Hamiltonian matrix elements, we have

$$
\left\langle\psi_{i}|H| \psi_{j}\right\rangle=8 \pi^{2} \int_{0}^{\infty} \int_{0}^{\infty} \int_{\left|r_{1}-r_{2}\right|}^{r_{1}+r_{2}} \psi_{i}^{*}\left(H \psi_{j}\right) r_{1} r_{2} r_{12} d r_{1} d r_{2} d r_{12}
$$

where 


$$
H_{i j}=\left[\begin{array}{cccc}
\left\langle\psi_{1}|H| \psi_{1}\right\rangle & \left\langle\psi_{1}|H| \psi_{2}\right\rangle & \left\langle\psi_{1}|H| \psi_{3}\right\rangle & \ldots \\
\left\langle\psi_{2}|H| \psi_{1}\right\rangle & \left\langle\psi_{2}|H| \psi_{2}\right\rangle & \left\langle\psi_{2}|H| \psi_{3}\right\rangle & \ldots \\
\left\langle\psi_{3}|H| \psi_{1}\right\rangle & \left\langle\psi_{3}|H| \psi_{2}\right\rangle & \left\langle\psi_{3}|H| \psi_{3}\right\rangle & \ldots \\
\vdots & \vdots & \vdots & \ddots
\end{array}\right] .
$$

The eigensystem is

$$
H \psi=E \psi .
$$

Muliplying by both sides by $\psi^{*}$ and integrating over all space the energy eigenvalue can be expressed as

$$
E=\frac{\int \psi^{*}(H \psi) d V}{\int \psi^{*} \psi d V}=\frac{\sum c_{i}^{*} c_{j} H_{i j}}{\sum c_{i}^{*} c_{j} \Delta_{i j}}
$$

where $\psi=\sum c_{i} \psi_{i}$ and the sigma means we sum over any repeated index ([18] p.187). Thus,

$$
E \sum c_{i}^{*} c_{j} \Delta_{i j}=\sum c_{i}^{*} c_{j} H_{i j}
$$

To find the values of $c_{i}$ that make $E$ a minimum, we differentiate with respect to each $c_{k}$ :

$$
\frac{\partial E}{\partial c_{k}} \sum c_{i}^{*} c_{j} \Delta_{i j}+E \frac{\partial}{\partial c_{k}} \sum c_{i}^{*} c_{j} \Delta_{i j}=\frac{\partial}{\partial c_{k}} \sum c_{i}^{*} c_{j} H_{i j}
$$

The condition for a minimum is that $\frac{\partial E}{\partial c_{k}}=0$ for all $k=1,2,3 \ldots$, which leads to the set of equations to diagonalize,

$$
\sum_{i} c_{i}\left(H_{i j}-\Delta_{i j} E\right)=0 .
$$

For smaller basis sets, we may also use the fact that for a non-trivial solution it is 
necessary the determinant of the coefficients to vanish,

$$
\operatorname{det}\left[H_{i j}-\Delta_{i j} E\right]=0
$$

This equation leads to a large polynomial in E, where the lowest value energy, $E_{\text {min }}$, corresponds to the ground-state energy. Plugging $E_{\text {min }}$ back into $\sum_{i} c_{i}\left(H_{i j}-\Delta_{i j} E\right)=$ 0 , one can determine the coefficients $c_{i}$. 


\section{Section 2}

\section{Photodetachment}

Starting in the 1940's, Chandrasekhar expanded on the study of the continuous absorption coefficient of the negative hydrogen ion initiated by Jen [19]. The key problem is to evaluate the (length gauge) dipole transition matrix element $\mu_{z}=$ $\int \psi_{d}^{*}\left(z_{1}+z_{2}\right) \psi_{c} d \tau[20]$, where $\psi_{d}$ denotes the wave function of the ground state of the ion and $\psi_{c}$ is a continuum state wave function correspond to the outgoing electron. Once the dipole transition matrix elements are evaluated, the standard length formula for the atomic absorption coefficient is

$$
\sigma=\left(6.812 \times 10^{-20} \mathrm{~cm}^{2}\right) k\left(k^{2}+2 I\right)\left|\mu_{z}\right|^{2}
$$

where $I$ denotes the electron affinity and $k$ is the momentum of the ejected electron, with all quantities in atomic units [21][22][13]. Evaluating $\mu_{z}$ for wave functions $\psi_{d}$ of the Hylleraas form [1] has historically been done via numerical integration. We present an analytical integration method for such wave functions herein. Although this formalism was developed for the specific problem of photodetachment, it is likely to find utility in other problems that calculate dipole transitions involving single

photons or laser fields. In order to evaluate $\mu_{z}$, we shall assume $\psi_{d}$ to be a wave function of the Hylleraas form, and $\psi_{c}$ a plane wave representation of the outgoing electron and a 1s state for the remaining electron:

$$
\psi_{c}=\frac{1}{\sqrt{2 \pi}}\left(e^{i k z_{1}-r_{2}}+e^{i k z_{2}-r_{1}}\right)
$$


where we have chosen the z-axis of the coordinate system to correspond to the propagation direction of the outgoing electron.

\section{Definitions}

The Hylleraas coordinate system [1] utilizes coordinates $s=r_{1}+r_{2}, t=r_{1}-r_{2}$, $u=r_{12} \equiv\left|\mathbf{r}_{1}-\mathbf{r}_{2}\right|$, and one builds wavefunction of the type

$$
\psi_{d}\left(r_{1}, r_{2}, r_{12}\right)=\frac{1}{\sqrt{2}}\left(1-\hat{P}_{12}\right) e^{-\alpha r_{1}-\beta r_{2}-\gamma r_{12}} \sum_{l, m, n} c_{l m n} s^{l} t^{2 m} u^{n} .
$$

The action of the Hamiltonian on Hylleraas wavefunctions leads to generic radial integrals of the type found by Calais and Lowdin [23], Sack, Roothaan and Kolos [24], and more recently Bhatia [25][26]

$$
\Gamma_{l m n}(\alpha, \beta, \gamma)=\int_{0}^{\infty} d r_{1} \int_{0}^{\infty} d r_{2} \int_{\left|r_{1}-r_{2}\right|}^{r_{1}+r_{2}} d r_{12} r_{1}^{l} r_{2}^{m} r_{12}^{n} e^{-\alpha r_{1}-\beta r_{2}-\gamma r_{12}}
$$

where $\alpha, \beta, \gamma$ are non-negative real numbers. We also assume that $l, m, n$ are nonnegative integers. A compact analytical formula for Eqn. (50) has been provided by Harris, Frolov and Smith [2],

$\Gamma_{l m n}(\alpha, \beta, \gamma)=2 l ! m ! n ! \sum_{l^{\prime}=0}^{l} \sum_{m^{\prime}=0}^{m} \sum_{n^{\prime}=0}^{n} \frac{\left(\begin{array}{c}m-m^{\prime}+l^{\prime} \\ l^{\prime}\end{array}\right)\left(\begin{array}{c}l-l^{\prime}+n^{\prime} \\ n^{\prime}\end{array}\right)\left(\begin{array}{c}n-n^{\prime}+m^{\prime} \\ m^{\prime}\end{array}\right)}{(\alpha+\beta)^{m-m^{\prime}+l^{\prime}+1}(\alpha+\gamma)^{l-l^{\prime}+n^{\prime}+1}(\beta+\gamma)^{n-n^{\prime}+m^{\prime}+1}}$. 
Integrals $\Lambda_{l m n}(\alpha, \beta, \gamma ; k)$

In the calculations of electric dipole moments elements using Hylleraas wavefunctions, one encounters integrals of the form

$\Lambda_{l m n}(\alpha, \beta, \gamma ; k)=\frac{1}{8 \pi^{2}} \iint e^{-\alpha r_{1}-\beta r_{2}-\gamma r_{12}} r_{1}^{l} r_{2}^{m} r_{12}^{n}\left(z_{1}+z_{2}\right)\left(e^{i k z_{1}-r_{2}}+e^{i k z_{2}-r_{1}}\right) d^{3} x_{1} d^{3} x_{2}$

where we have dropped the normalization constant $1 / \sqrt{2 \pi}$ from $\psi_{c}$. This is the integral we must evaluate. The approach we adopt, however, may well be generalizable to a wider set of single photoionization and laser problems where one can find integrals that take on similar forms to Eqn. (52) [27][28] [29][30]. Eqn. (52) can be written in the form

$$
\begin{aligned}
\Lambda_{l m n}(\alpha, \beta, \gamma ; k) & =\frac{1}{8 \pi^{2}} \iint e^{-\alpha r_{1}-\beta r_{2}-\gamma r_{12}} r_{1}^{l} r_{2}^{m} r_{12}^{n} z_{1} e^{i k z_{1}-r_{2}} d^{3} x_{1} d^{3} x_{2} \\
& +\frac{1}{8 \pi^{2}} \iint e^{-\alpha r_{1}-\beta r_{2}-\gamma r_{12}} r_{1}^{l} r_{2}^{m} r_{12}^{n} z_{1} e^{i k z_{2}-r_{1}} d^{3} x_{1} d^{3} x_{2} \\
& +\frac{1}{8 \pi^{2}} \iint e^{-\alpha r_{1}-\beta r_{2}-\gamma r_{12}} r_{1}^{l} r_{2}^{m} r_{12}^{n} z_{2} e^{i k z_{1}-r_{2}} d^{3} x_{1} d^{3} x_{2} \\
& +\frac{1}{8 \pi^{2}} \iint e^{-\alpha r_{1}-\beta r_{2}-\gamma r_{12}} r_{1}^{l} r_{2}^{m} r_{12}^{n} z_{2} e^{i k z_{2}-r_{1}} d^{3} x_{1} d^{3} x_{2} .
\end{aligned}
$$

We will break this into smaller pieces by defining each term in the previous equation as

$$
\begin{aligned}
\Lambda_{l m n}(\alpha, \beta, \gamma ; k) & =\Lambda_{l m n}^{11}(\alpha, \beta, \gamma ; k) \\
& +\Lambda_{l m n}^{12}(\alpha, \beta, \gamma ; k) \\
& +\Lambda_{l m n}^{21}(\alpha, \beta, \gamma ; k) \\
& +\Lambda_{l m n}^{22}(\alpha, \beta, \gamma ; k) .
\end{aligned}
$$

Integrals $\Lambda_{l m n}^{11}(\alpha, \beta, \gamma ; k)$

We first focus our attention on the term, 


$$
\Lambda_{l m n}^{11}(\alpha, \beta, \gamma ; k)=\frac{1}{8 \pi^{2}} \iint e^{-\alpha r_{1}-\beta r_{2}-\gamma r_{12}} r_{1}^{l} r_{2}^{m} r_{12}^{n} z_{1} e^{i k z_{1}-r_{2}} d^{3} x_{1} d^{3} x_{2}
$$

Using spherical harmonic expansions [31];

$$
z=r \sqrt{\frac{4 \pi}{3}} Y_{10}(\theta, \phi)
$$

and

$$
e^{i k r \cos \theta}=4 \pi \sum_{l=0}^{\infty} \sum_{m=-l}^{l} i^{l} j_{l}(k r) Y_{l m}\left(\theta_{r}, \phi_{r}\right) Y_{l m}^{*}\left(\theta_{k}, \phi_{k}\right),
$$

it can be shown that

$$
\begin{aligned}
\Lambda_{l m n}^{11}(\alpha, \beta, \gamma ; k)= & \frac{1}{2 \pi} \sqrt{\frac{4 \pi}{3}} \iint e^{-\alpha r_{1}-\beta r_{2}-\gamma r_{12}} r_{1}^{l} r_{2}^{m} r_{12}^{n} r_{1} Y_{10}\left(\theta_{1}, \phi_{1}\right) \\
& \times e^{-r_{2}} \sum_{L=0}^{\infty} \sum_{m=-L}^{L} i^{L} j_{L}\left(k r_{1}\right) Y_{L M}\left(\theta_{1}, \phi_{1}\right) Y_{L M}^{*}\left(\theta_{k}, \phi_{k}\right) d^{3} x_{1} d^{3} x_{2} .
\end{aligned}
$$

By expressing the volume element in terms of the solid angle $d \Omega_{12}$ (See Calais [23])

$$
\begin{gathered}
d^{3} x_{1} d^{3} x_{2}=r_{1}^{2} \sin \theta_{1} d r_{1} d \theta_{1} d \phi_{1} r_{2}^{2} \sin \theta_{2} d r_{2} d \theta_{2} d \phi_{2} \\
=r_{1}^{2} \sin \theta_{1} d r_{1} d \theta_{1} d \phi_{1} r_{2}^{2} \sin \theta_{12} d r_{2} d \theta_{12} d \phi_{12} \\
=r_{1}^{2} d r_{1} d \Omega_{1} r_{2}^{2} d r_{2} d \Omega_{12}
\end{gathered}
$$

we may more readily evaluate the integral. With this transformation, one finds

$$
\begin{aligned}
& \Lambda_{l m n}^{11}(\alpha, \beta, \gamma ; k)= \frac{1}{2 \pi} \sqrt{\frac{4 \pi}{3}} \sum_{L=0}^{\infty} \sum_{m=-L}^{L} i^{L} Y_{L M}^{*}\left(\theta_{k}, \phi_{k}\right) \\
& \times \iint e^{-\alpha r_{1}-\beta r_{2}-\gamma r_{12}} r_{1}^{l+3} r_{2}^{m+2} r_{12}^{n} e^{-r_{2}} j_{L}\left(k r_{1}\right) d r_{1} d r_{2} \\
& \times \int Y_{10}\left(\theta_{1}, \phi_{1}\right) Y_{L M}\left(\theta_{1}, \phi_{1}\right) d \Omega_{1} \int d \Omega_{12} .
\end{aligned}
$$


Using orthogonality,

$$
\begin{aligned}
& \Lambda_{l m n}^{11}(\alpha, \beta, \gamma ; k)= \frac{1}{2 \pi} \sqrt{\frac{4 \pi}{3}} \sum_{L=0}^{\infty} \sum_{m=-L}^{L} i^{L} Y_{L M}^{*}\left(\theta_{k}, \phi_{k}\right) \\
& \times \iint e^{-\alpha r_{1}-\beta r_{2}-\gamma r_{12}} r_{1}^{l+3} r_{2}^{m+2} r_{12}^{n} e^{-r_{2}} j_{L}\left(k r_{1}\right) d r_{1} d r_{2} \\
& \times \delta_{L 1} \delta_{M 0} \int d \Omega_{12}
\end{aligned}
$$

or

$$
\Lambda_{l m n}^{11}(\alpha, \beta, \gamma ; k)=\frac{1}{2 \pi} i \iint e^{-\alpha r_{1}-\beta r_{2}-\gamma r_{12}} r_{1}^{l+3} r_{2}^{m+2} r_{12}^{n} e^{-r_{2}} j_{1}\left(k r_{1}\right) d r_{1} d r_{2} \int d \Omega_{12}
$$

The last integral can be written out in terms of spherical angles,

$$
\Lambda_{l m n}^{11}(\alpha, \beta, \gamma ; k)=\frac{1}{2 \pi} i \iint e^{-\alpha r_{1}-\beta r_{2}-\gamma r_{12}} r_{1}^{l+3} r_{2}^{m+2} r_{12}^{n} e^{-r_{2}} j_{1}\left(k r_{1}\right) d r_{1} d r_{2} \int \sin \theta_{12} d \theta_{12} d \phi_{12} .
$$

Now we can convert angles into magnitudes by law of cosines

$$
\cos \theta_{12}=\frac{r_{1}^{2}+r_{2}^{2}-r_{12}^{2}}{2 r_{1} r_{2}}
$$

This gets one into a function of the basis $\left(r_{1}, r_{2}, r_{12}\right)$ by noting that

$$
r_{12} d r_{12}=r_{1} r_{2} \sin \theta_{12} d \theta_{12}
$$

In order to determine the limits, we note that when $\theta_{12}=0$ then $\cos \theta_{12}=1$. Therefore we have $r_{12}^{2}=r_{1}^{2}+r_{2}^{2}-2 r_{1} r_{2}$ and we find $r_{12}=\left|r_{1}-r_{2}\right|$. Also, when $\theta_{12}=\pi$ then $\cos \theta_{12}=-1$. Therefore we have $r_{12}^{2}=r_{1}^{2}+r_{2}^{2}+2 r_{1} r_{2}$ and $r_{12}=r_{1}+r_{2}$. Using Eqn. (65) with the correct limits of integration and after evaluating the $\phi_{12}$ integral, we 
find the result,

$$
\Lambda_{l m n}^{11}(\alpha, \beta, \gamma ; k)=i \iiint_{\left|r_{1}-r_{2}\right|}^{r_{1}+r_{2}} e^{-\alpha r_{1}-(\beta+1) r_{2}-\gamma r_{12}} r_{1}^{l+2} r_{2}^{m+1} r_{12}^{n+1} j_{1}\left(k r_{1}\right) d r_{1} d r_{2} d r_{12}
$$

Integrals $\Lambda_{l m n}^{12}(\alpha, \beta, \gamma ; k)$

The second term is

$$
\Lambda_{l m n}^{12}(\alpha, \beta, \gamma ; k)=\frac{1}{8 \pi^{2}} \iint e^{-\alpha r_{1}-\beta r_{2}-\gamma r_{12}} r_{1}^{l} r_{2}^{m} r_{12}^{n} z_{1} e^{i k z_{2}-r_{1}} d^{3} x_{1} d^{3} x_{2}
$$

or

$$
\begin{gathered}
\Lambda_{l m n}^{12}(\alpha, \beta, \gamma ; k)= \\
\frac{1}{2 \pi} \sqrt{\frac{4 \pi}{3}} \iint e^{-\alpha r_{1}-\beta r_{2}-\gamma r_{12}} r_{1}^{l} r_{2}^{m} r_{12}^{n} r_{1} Y_{10}\left(\theta_{1}, \phi_{1}\right) \\
e^{-r_{1}} \sum_{L=0}^{\infty} \sum_{m=-L}^{L} i^{L} j_{L}\left(k r_{2}\right) Y_{L M}\left(\theta_{2}, \phi_{2}\right) Y_{L M}^{*}\left(\theta_{k}, \phi_{k}\right) d^{3} x_{1} d^{3} x_{2}
\end{gathered}
$$

Using the expansion [32],

$$
\begin{gathered}
Y_{10}\left(\theta_{1}, \phi_{1}\right)=\sum_{M^{\prime}=-1}^{1}\left[D_{0 M^{\prime}}^{(1)}\left(0, \theta_{2}, \phi_{2}\right)\right]^{*} Y_{1 M^{\prime}}\left(\theta_{12}, \phi_{12}\right) \\
=\sum_{M^{\prime}=-1}^{1}\left[\sqrt{\frac{4 \pi}{3}} Y_{1 M^{\prime}}\left(, \theta_{2}, \phi_{2}\right)\right]^{*} Y_{1 M^{\prime}}\left(\theta_{12}, \phi_{12}\right)
\end{gathered}
$$

one finds

$$
\begin{aligned}
\Lambda_{l m n}^{12}(\alpha, \beta, \gamma ; k)= & \frac{1}{2 \pi} \frac{4 \pi}{3} \sum_{L=0}^{\infty} \sum_{M=-L}^{L} \sum_{M^{\prime}=-1}^{1} i^{L} Y_{L M}^{*}\left(\theta_{k}, \phi_{k}\right) \\
& \times \iint e^{-\alpha r_{1}-\beta r_{2}-\gamma r_{12}} r_{1}^{l+3} r_{2}^{m+2} r_{12}^{n} e^{-r_{1}} j_{L}\left(k r_{2}\right) d r_{1} d r_{2} \\
\times & \int Y_{1 M^{\prime}}^{*}\left(\theta_{2}, \phi_{2}\right) Y_{L M}\left(\theta_{2}, \phi_{2}\right) d \Omega_{2} \int Y_{1 M^{\prime}}\left(\theta_{12}, \phi_{12}\right) d \Omega_{12}
\end{aligned}
$$




$$
\begin{gathered}
=\quad \frac{1}{2 \pi} \frac{4 \pi}{3} \sum_{L=0}^{\infty} \sum_{M=-L}^{L} \sum_{M^{\prime}=-1}^{1} i^{L} Y_{L M}^{*}\left(\theta_{k}, \phi_{k}\right) r_{2} \\
\times \iint e^{-\alpha r_{1}-\beta r_{2}-\gamma r_{12}} r_{1}^{l+3} r_{2}^{m+2} r_{12}^{n} e^{-r_{1}} j_{L}\left(k r_{2}\right) d r_{1} d \\
\times \delta_{L 1} \delta_{M^{\prime} M} \int Y_{1 M^{\prime}}\left(\theta_{12}, \phi_{12}\right) d \Omega_{12} \\
=\quad \frac{1}{2 \pi} \frac{4 \pi}{3} \sum_{M=-1}^{1} i Y_{1 M}^{*}\left(\theta_{k}, \phi_{k}\right) \\
\times \iint e^{-\alpha r_{1}-\beta r_{2}-\gamma r_{12}} r_{1}^{l+3} r_{2}^{m+2} r_{12}^{n} e^{-r_{1}} j_{1}\left(k r_{2}\right) d r_{1} d r_{2} \\
\times \int Y_{1 M}\left(\theta_{12}, \phi_{12}\right) d \Omega_{12} .
\end{gathered}
$$

Here we note that $\int e^{i \phi} d \phi=0$, which means only $M=0$ will be the non-zero term in the summation. Thus, we have

$$
\begin{array}{cc}
\Lambda_{l m n}^{12}(\alpha, \beta, \gamma ; k)= & \frac{1}{2 \pi} \frac{4 \pi}{3} i Y_{10}^{*}\left(\theta_{k}, \phi_{k}\right) \\
\times \iint e^{-\alpha r_{1}-\beta r_{2}-\gamma r_{12}} r_{1}^{l+3} r_{2}^{m+2} r_{12}^{n} e^{-r_{1}} j_{1}\left(k r_{2}\right) d r_{1} d r_{2} \\
\times \int Y_{10}\left(\theta_{12}, \phi_{12}\right) d \Omega_{12} .
\end{array}
$$

Expanding $Y_{10}\left(\theta_{12}, \phi_{12}\right)$ in terms of trigonometric functions leads to

$$
\begin{gathered}
\Lambda_{l m n}^{12}(\alpha, \beta, \gamma ; k)=\frac{1}{2 \pi} i \iint e^{-\alpha r_{1}-\beta r_{2}-\gamma r_{12}} r_{1}^{l+3} r_{2}^{m+2} r_{12}^{n} e^{-r_{1}} j_{1}\left(k r_{2}\right) d r_{1} d r_{2} \\
\times \iint \cos \theta_{12} \sin \theta_{12} d \theta_{12} d \phi_{12} .
\end{gathered}
$$

Applying Eqn. (64) and Eqn. (65) to the angle integral, we find

$$
\begin{gathered}
\Lambda_{l m n}^{12}(\alpha, \beta, \gamma ; k)=\frac{1}{2 \pi} i \iint e^{-\alpha r_{1}-\beta r_{2}-\gamma r_{12}} r_{1}^{l+3} r_{2}^{m+2} r_{12}^{n} e^{-r_{1}} j_{1}\left(k r_{2}\right) d r_{1} d r_{2} \\
\times \int \frac{r_{1}^{2}+r_{2}^{2}-r_{12}^{2}}{2 r_{1} r_{2}} \frac{r_{12}}{r_{1} r_{2}} d r_{12} \int d \phi_{12}
\end{gathered}
$$




$$
\begin{aligned}
& \Lambda_{l m n}^{12}(\alpha, \beta, \gamma ; k)=\quad \frac{1}{2} i \iiint e^{-(\alpha+1) r_{1}-\beta r_{2}-\gamma r_{12}} j_{1}\left(k r_{2}\right) \\
& \times\left(r_{1}^{l+3} r_{2}^{m} r_{12}^{n+1}+r_{1}^{l+1} r_{2}^{m+2} r_{12}^{n+1}-r_{1}^{l+1} r_{2}^{m} r_{12}^{n+3}\right) d r_{1} d r_{2} d r_{12} \text {. }
\end{aligned}
$$

The final two integrals $\Lambda_{l m n}^{21}(\alpha, \beta, \gamma ; k)$ and $\Lambda_{l m n}^{22}(\alpha, \beta, \gamma ; k)$ are essentially identical to the proceding integrals with $r_{1}$ and $r_{2}$ switched. Putting this all together leads to the result,

$$
\begin{gathered}
\Lambda_{l m n}(\alpha, \beta, \gamma ; k)=i \int_{0}^{\infty} d r_{1} \int_{0}^{\infty} d r_{2} \int_{\left|r_{1}-r_{2}\right|}^{r_{1}+r_{2}} d r_{12} j_{1}\left(k r_{1}\right) r_{1}^{l+2} r_{2}^{m+1} r_{12}^{n+1} e^{-\alpha r_{1}-(\beta+1) r_{2}-\gamma r_{12}} \\
+\frac{i}{2} \int_{0}^{\infty} d r_{1} \int_{0}^{\infty} d r_{2} \int_{\left|r_{1}-r_{2}\right|}^{r_{1}+r_{2}} d r_{12} j_{1}\left(k r_{1}\right)\left(r_{1}^{l} r_{2}^{m+3} r_{12}^{n+1}+r_{1}^{l+2} r_{2}^{m+1} r_{12}^{n+1}-r_{1}^{l} r_{2}^{m+1} r_{12}^{n+3}\right) \\
\times e^{-\alpha r_{1}-(\beta+1) r_{2}-\gamma r_{12}} \\
+\frac{i}{2} \int_{0}^{\infty} d r_{1} \int_{0}^{\infty} d r_{2} \int_{\left|r_{1}-r_{2}\right|}^{r_{1}+r_{2}} d r_{12} j_{1}\left(k r_{2}\right)\left(r_{1}^{l+3} r_{2}^{m} r_{12}^{n+1}+r_{1}^{l+1} r_{2}^{m+2} r_{12}^{n+1}-r_{1}^{l+1} r_{2}^{m} r_{12}^{n+3}\right) \\
\times e^{-(\alpha+1) r_{1}-\beta r_{2}-\gamma r_{12}} \\
+i \int_{0}^{\infty} d r_{1} \int_{0}^{\infty} d r_{2} \int_{\left|r_{1}-r_{2}\right|}^{r_{1}+r_{2}} d r_{12} j_{1}\left(k r_{2}\right) r_{1}^{l+1} r_{2}^{m+2} r_{12}^{n+1} e^{-(\alpha+1) r_{1}-\beta r_{2}-\gamma r_{12}},
\end{gathered}
$$

where $j_{1}(x)=\frac{\sin x}{x^{2}}-\frac{\cos x}{x}$ is the spherical Bessel function of the first kind.

Integrals $J_{L M N}(a, b, c ; k)$

The integrals that make up $\Lambda_{l m n}(\alpha, \beta, \gamma ; k)$ share many similarities with $\Gamma_{l m n}(\alpha, \beta, \gamma)$, with one additional function $j_{1}(k r)$. We follow the definition given by Frolov and Wardlaw [33],

$$
J_{L M N}(a, b, c ; k)=\int_{0}^{\infty} d r_{1} \int_{0}^{\infty} d r_{2} \int_{\left|r_{1}-r_{2}\right|}^{r_{1}+r_{2}} d r_{12} j_{1}\left(k r_{1}\right) r_{1}^{L} r_{2}^{M} r_{12}^{N} e^{-a r_{1}-b r_{2}-c r_{12}}
$$


where $a, b, c$ are non-negative real numbers. We also assume that $L, M, N$ are nonnegative integers.

Deriving an analytic formula for integrals of this type is the remaining goal in this study.

By switching the arguments in $J_{L M N}(a, b, c ; k)$ to $J_{L M N}(b, a, c ; k)$, Eq. (76) can be used in the cases where the spherical Bessel function has $k r_{2}$ as its argument. Using $J_{L M N}(a, b, c ; k)$, Eqn. (75) becomes

$$
\begin{gathered}
\Lambda_{l m n}(\alpha, \beta, \gamma ; k)=i J_{l+2, m+1, n+1}(\alpha, \beta+1, \gamma ; k) \\
+\frac{i}{2}\left[J_{l, m+3, n+1}(\alpha, \beta+1, \gamma ; k)+J_{l+2, m+1, n+1}(\alpha, \beta+1, \gamma ; k)-J_{l, m+1, n+3}(\alpha, \beta+1, \gamma ; k)\right] \\
+\frac{i}{2}\left[J_{m, l+3, n+1}(\beta, \alpha+1, \gamma ; k)+J_{m+2, l+1, n+1}(\beta, \alpha+1, \gamma ; k)-J_{m, l+1, n+3}(\beta, \alpha+1, \gamma ; k)\right] \\
+i J_{m+2, l+1, n+1}(\beta, \alpha+1, \gamma ; k),
\end{gathered}
$$

where we now assume that $l, m$, are non-negative integers and $n$ is an integer such that $n \geq-1$.

Frolov and Wardlaw [33] recently showed that,

$$
J_{000}(a, b, c ; k)=\frac{2}{b^{2}-c^{2}}[\mathcal{L}(a+c ; k)-\mathcal{L}(a+b ; k)]
$$

In this case, $\mathcal{L}(p ; k)$ turns out to be the Laplace transform of the spherical Bessel function $j_{1}(k r)$, although this procedure generalizes to any function by simply taking the Laplace transform of the specific function desired. In particular, for $j_{1}(k r)$ [34], [35],

$$
\mathcal{L}(p ; k)=\frac{1}{k^{2}}\left[k-p \tan ^{-1}\left(\frac{k}{p}\right)\right] .
$$




\section{General Approach}

Following the standard procedure of taking multiple derivatives within Eqn. (78) to obtain higher powers of $r_{1}, r_{2}$, and $r_{12}$ [23],

$$
J_{L M N}(a, b, c ; k)=(-1)^{L+M+N} \frac{\partial^{N}}{\partial c^{N}} \frac{\partial^{M}}{\partial b^{M}} \frac{\partial^{L}}{\partial a^{L}} J_{000}(a, b, c ; k) .
$$

Noting that only the $\mathcal{L}(p ; k)$ part of $J_{000}(a, b, c ; k)$ depends explicitly on $a$, we find that

$$
J_{L M N}(a, b, c ; k)=(-1)^{M+N} \frac{\partial^{N}}{\partial c^{N}} \frac{\partial^{M}}{\partial b^{M}}\left\{\frac{2}{b^{2}-c^{2}}\left[\mathcal{L}_{L}(a+c ; k)-\mathcal{L}_{L}(a+b ; k)\right]\right\}
$$

where

$$
\mathcal{L}_{L}(p ; k)=(-1)^{L} \frac{\partial^{L}}{\partial a^{L}} \mathcal{L}(p ; k)=(-1)^{L} \frac{\partial^{L}}{\partial a^{L}} \frac{1}{k^{2}}\left[k-p \tan ^{-1}\left(\frac{k}{p}\right)\right] .
$$

and $p=a+c$ or $a+b$. It can be shown that

$$
\mathcal{L}_{L}(p ; k)=\left\{\begin{array}{cc}
\frac{1}{k^{2}}\left[k-p \tan ^{-1}\left(\frac{k}{p}\right)\right] & L=0 \\
\frac{1}{k^{2}}\left[-\frac{k p}{p^{2}+k^{2}}+\tan ^{-1}\left(\frac{k}{p}\right)\right] & L=1 \\
\frac{i(L-2) !}{2 k^{2}}\left[\frac{p+i k L}{(p+i k)^{L}}-\frac{p-i k L}{(p-i k)^{L}}\right]= & \\
-\frac{(L-2) !}{k\left(k^{2}+p^{2}\right)^{L}} \sum_{j=0}^{L}{ }^{(2)}\left(\begin{array}{c}
L \\
j
\end{array}\right) \frac{j(L+1)}{j+1}(-1)^{j / 2} k^{j} p^{L-j} & L=2,3,4, \ldots \\
\frac{1}{3}(L+1) ! \frac{k}{p^{L+2}}{ }_{2} F_{1}\left(\frac{L+2}{2}, \frac{L+3}{2} ; \frac{5}{2} ;-\frac{k^{2}}{p^{2}}\right) & L \geq 0
\end{array},\right.
$$




$$
(-1)^{L} \frac{\partial^{L}}{\partial p^{L}} \mathfrak{L}\left(j_{l}(k r) ; p\right)=\frac{(L+l) ! \Gamma\left(\frac{1}{2}\right)}{2^{l+1} \Gamma(l+3 / 2)} \frac{k^{l}}{p^{l+L+1}} F\left(\frac{L+1+l}{2}, \frac{L+2+l}{2} ; l+\frac{3}{2},-\frac{k^{2}}{p^{2}}\right),
$$

where the "(2)" on the summation sign in the last line indicates steps of two. We have included Eqn. (84) (for $l$ not necessarily equal to 1 ) for completeness.

Next, we use the Leibniz product rule for multiple derivatives [36],

$$
\begin{gathered}
J_{L M N}(a, b, c ; k)=(-1)^{M+N} \sum_{N^{\prime}=0}^{N} \sum_{M^{\prime}=0}^{M}\left(\begin{array}{c}
N \\
N^{\prime}
\end{array}\right)\left(\begin{array}{c}
M \\
M^{\prime}
\end{array}\right) \frac{\partial^{N^{\prime}}}{\partial c^{N^{\prime}}} \frac{\partial^{M^{\prime}}}{\partial b^{M^{\prime}}} \frac{2}{b^{2}-c^{2}} \\
\times \frac{\partial^{N-N^{\prime}}}{\partial c^{N-N^{\prime}}} \frac{\partial^{M-M^{\prime}}}{\partial b^{M-M^{\prime}}}\left[\mathcal{L}_{L}(a+c ; k)-\mathcal{L}_{L}(a+b ; k)\right],
\end{gathered}
$$

and define the function

$$
F_{M N}(b, c)=(-1)^{M+N} \frac{\partial^{N}}{\partial c^{N}} \frac{\partial^{M}}{\partial b^{M}} \frac{2}{b^{2}-c^{2}} .
$$

It can be shown that

$$
F_{M N}(b, c)=2 M ! N ! \sum_{M^{\prime}=0}^{M} \sum_{N^{\prime}=0}^{N}(-1)^{N^{\prime}} \frac{\left(\begin{array}{c}
M-M^{\prime}+N^{\prime} \\
N^{\prime}
\end{array}\right)}{(b-c)^{M-M^{\prime}+N^{\prime}+1}} \frac{\left(\begin{array}{c}
N-N^{\prime}+M^{\prime} \\
M^{\prime}
\end{array}\right)}{(b+c)^{N-N^{\prime}+M^{\prime}+1}} .
$$

Thus, 


$$
\begin{aligned}
& \sum_{N^{\prime}=0}^{N} \sum_{M^{\prime}=0}^{M}\left(\begin{array}{c}
N \\
N^{\prime}
\end{array}\right)\left(\begin{array}{c}
M \\
M^{\prime}
\end{array}\right) F_{M^{\prime} N^{\prime}}(b, c)(-1)^{N-N^{\prime}+M-M^{\prime}} \frac{\partial^{N-N^{\prime}}}{\partial c^{N-N^{\prime}}} \frac{\partial^{M-M^{\prime}}}{\partial b^{M-M^{\prime}}} \mathcal{L}_{L}(a+c ; k) \\
& -\sum_{N^{\prime}=0}^{N} \sum_{M^{\prime}=0}^{M}\left(\begin{array}{c}
N \\
N^{\prime}
\end{array}\right)\left(\begin{array}{c}
M \\
M^{\prime}
\end{array}\right) F_{M^{\prime} N^{\prime}}(b, c)(-1)^{N-N^{\prime}+M-M^{\prime}} \frac{\partial^{N-N^{\prime}}}{\partial c^{N-N^{\prime}}} \frac{\partial^{M-M^{\prime}}}{\partial b^{M-M^{\prime}}} \mathcal{L}_{L}(a+b ; k) .
\end{aligned}
$$

Looking at the term $\frac{\partial^{M-M^{\prime}}}{\partial b^{M-M^{\prime}}} \mathcal{L}_{L}(a+c ; k)$, we note that $\mathcal{L}_{L}(a+c ; k)$ is not a function of b. Thus, this term is zero unless $M-M^{\prime}=0$. Likewise, for the term $\frac{\partial^{N-N^{\prime}}}{\partial c^{N-N^{\prime}}} \mathcal{L}_{L}(a+b ; k)$, we see that $N-N^{\prime}=0$. Therefore,

$$
\begin{aligned}
J_{L M N}(a, b, c ; k) & =\sum_{N^{\prime}=0}^{N}\left(\begin{array}{c}
N \\
N^{\prime}
\end{array}\right) F_{M N^{\prime}}(b, c)(-1)^{N-N^{\prime}} \frac{\partial^{N-N^{\prime}}}{\partial c^{N-N^{\prime}}} \mathcal{L}_{L}(a+c ; k) \\
& -\sum_{M^{\prime}=0}^{M}\left(\begin{array}{c}
M \\
M^{\prime}
\end{array}\right) F_{M^{\prime} N}(b, c)(-1)^{M-M^{\prime}} \frac{\partial^{M-M^{\prime}}}{\partial b^{M-M^{\prime}}} \mathcal{L}_{L}(a+b ; k) .
\end{aligned}
$$

This finally produces the formula desired for this study

$$
\begin{aligned}
J_{L M N}(a, b, c ; k)= & \sum_{N^{\prime}=0}^{N}\left(\begin{array}{c}
N \\
N^{\prime}
\end{array}\right) F_{M N^{\prime}}(b, c) \mathcal{L}_{L+N-N^{\prime}}(a+c ; k) \\
& -\sum_{M^{\prime}=0}^{M}\left(\begin{array}{c}
M \\
M^{\prime}
\end{array}\right) F_{M^{\prime} N}(b, c) \mathcal{L}_{L+M-M^{\prime}}(a+b ; k) .
\end{aligned}
$$

This procedure can be generalized to any integral of the form 


$$
J_{L M N}(a, b, c ; k)=\int_{0}^{\infty} d r_{1} \int_{0}^{\infty} d r_{2} \int_{\left|r_{1}-r_{2}\right|}^{r_{1}+r_{2}} d r_{12} f\left(k r_{1}\right) r_{1}^{L} r_{2}^{M} r_{12}^{N} e^{-a r_{1}-b r_{2}-c r_{12}}
$$

as long as one can find the Laplace transform of $f\left(k r_{1}\right)$.

\section{Conclusion}

We have confirmed that by using Hylleraas coordinates, the three body system of helium could be solved via the variational principle, and it generates very accurate results for ground state energies and wavefunctions, which are the starting points for calculations of photodetachment. We then developed an approach that is successful at obtaining closed form analytical formulas for three body integrals containing any function $f\left(k r_{1}\right)$, provided one can find the Laplace transform of the function. Our interest in obtaining these formulas was the application to calculation of photodetachment in three-body atoms. However, such integrals are of interest in many problems in the areas of atomic and nuclear physics. While solutions to these integrals have heretofore been evaluated numerically, the computational load is greatly reduced with an analytical result. 


\section{References}

[1] E. A. Hyllerass. Neue berechnung der energie des heliums im grundzustande, sowie des tiefsten terms von ortho-helium. Z. Physik, 54(347), 1929.

[2] F. E. Harris, A. M. Frolov, and V. H. Smith Jr. Singular and nonsingular threebody integrals for exponential wave functions. J. Chem. Phys., 121(13), 2004.

[3] David J. Griffiths. Introduction to Quantum Mechanics. Pearson Prentice Hall, p. 2992005.

[4] P.J. Linstrom and W.G. Mallard. NIST Chemistry WebBook, NIST Standard Reference Database. National Institute of Standards and Techology, http://webook.nist.gov, retrieved September 29, 2015.

[5] R. Whitnell R. Cranall and R. Bettega. Exactly soluble two-eletron atomic model. Am. J. Phys, 52, 19856.

[6] Jack C. Straton. Reduded form for the general-state multicenter integral form an integro-differential transform. Phys. Rev. A, 41(71), 1990.

[7] Jack C. Straton. Analytically reduced form of multicenter integrals from guassian transforms. Phy. Rev. A, 39(1676), 1989.

[8] Jack C. Straton. Fourier transform of the multicenter product of 1s hydrogenic orbitals and coulomb or yukawa potentials and the analytically reduced form of subsequent integrals that include plane waves. Phy. Rev. A, 39(5062), 1989.

[9] Jack C. Straton. Reduced form for coulomb-wave multicenter integrals. Phy. Rev. A, 42(307), 1990.

[10] Jackson. Classical Electrodynamics. John Wiley, p.111, 1999. 
[11] J. J. Gibbons J. H. Bartlett and C. G. Dunn. The normal helium atom. Phys. Rev., 47(679), 1935.

[12] Per-Olov Lowdin and Lajos Redei. Combined use of the methods of superposition of configurations and correlation factor on the ground states of the helium-like oons. Phys. Rev., 752, 114.

[13] R. E. Williamson. The negative hydrogen ion and its absorption cefficient. Astrophys J., 96(438), 1942.

[14] B.H. Bransden and C.J. Joachain. Physics of Atoms and Molecules. Pearson Education limited, Essex, p. 3322003.

[15] C. L. Pekeris. Ground state of two-electorn atoms. Phys. Rev., 112(5), 1958.

[16] C. L. Pekeris. 1 1s, 2 2s, and 2 3s states of h- and of he. Phys. Rev., 126(4), 1962.

[17] Morse and Feshback. Methods of Theoreical Physics. McGraw-Hill, p. 1731, 1953.

[18] Linus Pauling and E. Bright Wilson Jr. Introduction to Quantum Mechanics. Dover, p. 187, 1963.

[19] C. K. Jen. The continuous electron affinity spectrum of hydrogen. Phys. Rev., 43:540-547, Apr 1933. doi: 10.1103/PhysRev.43.540. URL http://link.aps.org/doi/10.1103/PhysRev.43.540.

[20] A. M. Frolov and D. M. Wardlaw. Three-particle integrals with bessel functions. Physics of Atomic Nuclei, 77(2):175-184, 2014.

[21] S. Chandrasekhar. On the continuous absorption coefficient of the negative hydrogen ion. Astrophys J., 102(223-231), 1945. 
[22] S. Geltman. The bound-free absorption coefficient of the hydrogen negarive ion. Astrophys J., 136(935), 1962.

[23] Jean-Louis Calais and Per-Olov Lowdin. A simple method of treating atomic integrlas containing funcitons of r12. J. Mol. Spectrosc., 8(203-211), 1962.

[24] R. A. Sack, C. C. J. Roothaan, and W. Kolos. Recursive evaluation of some atomic integrals. J. Math Phys., 1093(8), 1967.

[25] A. K. Bhatia. Properties of the ground state of the hydrogen molecular ion. Phys Rev A, 1998.

[26] A. K. Bhatia and Richard J. Drachman. Polarizability of the ground state of the hydrogen molecular ion. Phys. Rev. A, 59(1), 1998.

[27] C. Sinha and A. K. Bhatia. Laser-assisted free-free transition in electron-atom colisions. Phys. Rev. A, 83(Eqn. 9a), 2011.

[28] A. K. Bhatia and C. Sinha. Free-free transitions of the e-h system inside a dense plasma irradiated by a laser field at very low incident-electron energies. Phys Rev A, 87(Eqn. 18), 2012.

[29] Sougata Mukhopadhyay and C. Sinha. Formation of antihydrogen in an antiproton-positron debye plasma in the presence of an external laser field. Phy. Rev. A, 88(Eqn. 15), 2013.

[30] S. Bivona, R. Burlon, and C. Leone. Controlling laser assisted radiative recombination with few-cycle laser pulses. Laser Phys. Lett., 4(p.44-49 Eqn. 5), 2007.

[31] Charles J. Joachain. Quantum Collision Theory. Elsevier Science Ltd, p. 671, 1984. 
[32] E. Ley-Koo and Carlos F. Bunge. General evaluation of atomic electron-repulsion integrals in orbital methods without using a series representation for r12. Phys. Rev. A, 40(3), 1989.

[33] A. M. Frolov and D. M. Wardlaw. Three-particle integrals with spherical bessel and neumann functions and photodetachment of the negatively charged hydrogen ions. J. Math. Chem., 2015.

[34] I. S. Gradshteyn and I. M. Ryzhik. Table of Integrals, Series, and Products 5ed. Number 6.621.1(4). Academic, New York, 1994.

[35] I. S. Gradshteyn and I. M. Ryzhik. Table of Integrals, Series, and Products. Number 6.621.1(4). Academic, New York, 1994.

[36] M. Abramowitz and I. A. Stegun. Handbook of Mathematical Functions with Formulas, Graphs, and Mathematical Tables. Number p. 12, No. 3.3.8. National Bureau of Standards, Applied mathematics series, 1970. 


\section{Appendix}

\section{Derivation of the Hamiltonian in Hylleraas Coordinates}

We start with the Hamiltonian

$$
H=-\frac{1}{2} \nabla_{r_{1}}^{2}-\frac{1}{2} \nabla_{r_{2}}^{2}-\left(\frac{Z}{r_{1}}+\frac{Z}{r_{1}}-\frac{1}{r_{12}}\right)
$$

The Laplacian is defined as

$$
\begin{aligned}
& \nabla_{r_{1}}^{2}=\frac{\partial^{2}}{\partial x_{1}^{2}}+\frac{\partial^{2}}{\partial y_{1}^{2}}+\frac{\partial^{2}}{\partial z_{1}^{2}} \\
& \nabla_{r_{2}}^{2}=\frac{\partial^{2}}{\partial x_{2}^{2}}+\frac{\partial^{2}}{\partial y_{2}^{2}}+\frac{\partial^{2}}{\partial z_{2}^{2}} .
\end{aligned}
$$

We would like to transform into coordinates

$$
\begin{gathered}
r_{1}^{2}=x_{1}^{2}+y_{1}^{2}+z_{1}^{2} \\
r_{2}^{2}=x_{2}^{2}+y_{2}^{2}+z_{2}^{2} \\
r_{12}^{2}=\left(x_{2}-x_{1}\right)^{2}+\left(y_{2}-y_{1}\right)^{2}+\left(z_{2}-z_{1}\right)^{2} .
\end{gathered}
$$

We start with the chain rule

$$
\frac{\partial \psi}{\partial x_{1}}=\frac{\partial \psi}{\partial r_{1}} \frac{\partial r_{1}}{\partial x_{1}}+\frac{\partial \psi}{\partial r_{2}} \frac{\partial r_{2}}{\partial x_{1}}+\frac{\partial \psi}{\partial r_{12}} \frac{\partial r_{12}}{\partial x_{1}}
$$

The three derivatives we need are:

$$
\begin{gathered}
\frac{\partial r_{1}}{\partial x_{1}}=\frac{1}{2}\left(x_{1}^{2}+y_{1}^{2}+z_{1}^{2}\right)^{-1 / 2} 2 x_{1}=\frac{x_{1}}{r_{1}}, \\
\frac{\partial r_{2}}{\partial x_{1}}=0
\end{gathered}
$$




$$
\frac{\partial r_{12}}{\partial x_{1}}=\frac{1}{2}\left(\left(x_{2}-x_{1}\right)^{2}+\left(y_{2}-y_{1}\right)+\left(z_{2}-z_{1}\right)^{2}\right)^{-1 / 2} 2\left(x_{2}-x_{1}\right)(-1)=-\frac{x_{2}-x_{1}}{r_{12}}
$$

Thus,

$$
\frac{\partial \psi}{\partial x_{1}}=\frac{\partial \psi}{\partial r_{1}} \frac{x_{1}}{r_{1}}+\frac{\partial \psi}{\partial r_{2}} 0+\frac{\partial \psi}{\partial r_{12}}\left(-\frac{x_{2}-x_{1}}{r_{12}}\right)
$$

However, we still need the second derivative. Applying the chain rule once more, we find

$$
\frac{\partial^{2} \psi}{\partial x_{1}^{2}}=\frac{\partial \psi}{\partial r_{1}} \frac{\partial}{\partial x_{1}}\left(\frac{x_{1}}{r_{1}}\right)+\frac{\partial^{2} \psi}{\partial x_{1} d r_{1}} \frac{x_{1}}{r_{1}}+\frac{\partial \psi}{\partial r_{12}} \frac{\partial}{\partial x_{1}}\left(-\frac{x_{2}-x_{1}}{r_{12}}\right)+\frac{\partial^{2} \psi}{\partial x_{1} d r_{12}}\left(-\frac{x_{2}-x_{1}}{r_{12}}\right)
$$

The derivatives we need are as follows:

$$
\begin{gathered}
\frac{\partial}{\partial x_{1}}\left(\frac{x_{1}}{r_{1}}\right)=\frac{1}{r_{1}}+x_{1}\left[-\frac{1}{2}\left(x_{1}^{2}+y_{1}^{2}+z_{1}^{2}\right)^{-3 / 2} 2 x_{1}\right]=\frac{1}{r_{1}}-\frac{x_{1}^{2}}{r_{1}^{3}}, \\
\frac{\partial}{\partial x_{1}}\left(-\frac{x_{2}-x_{1}}{r_{12}}\right)= \\
\times\left[-\frac{1}{2}\left(\left(x_{2}-x_{1}\right)^{2}+\left(y_{2}-y_{1}\right)^{2}+\left(z_{2}-z_{1}\right)^{2}\right)^{-3 / 2} 2\left(x_{2}-x_{1}\right)(-1)\right] \\
=\frac{1}{r_{12}}-\frac{\left(x_{2}-x_{1}\right)^{2}}{r_{12}^{3}}, \\
\frac{\partial^{2} \psi}{\partial x_{1} d r_{1}}=\frac{\partial^{2} \psi}{\partial r_{1}^{2}} \frac{\partial r_{1}}{\partial x_{1}}+\frac{\partial^{2} \psi}{\partial r_{2} \partial r_{1}} \frac{\partial r_{2}}{\partial x_{1}}+\frac{\partial^{2} \psi}{\partial r_{12} \partial r_{1}} \frac{\partial r_{12}}{\partial x_{1}} \\
=\frac{\partial^{2} \psi}{\partial r_{1}^{2}} \frac{x_{1}}{r_{1}}+\frac{\partial^{2} \psi}{\partial r_{2} \partial r_{1}} 0+\frac{\partial^{2} \psi}{\partial r_{12} \partial r_{1}}\left(-\frac{x_{2}-x_{1}}{r_{12}}\right)
\end{gathered}
$$




$$
\begin{aligned}
\frac{\partial^{2} \psi}{\partial x_{1} \partial r_{12}} & =\frac{\partial^{2} \psi}{\partial r_{1} \partial r_{12}} \frac{\partial r_{1}}{\partial x_{1}}+\frac{\partial^{2} \psi}{\partial r_{2} \partial r_{12}} \frac{\partial r_{2}}{\partial x_{1}}+\frac{\partial^{2}}{\partial r_{12}^{2}} \frac{\partial r_{12}}{\partial x_{1}} \\
& =\frac{\partial^{2} \psi}{\partial r_{1} \partial r_{12}} \frac{x_{1}}{r_{1}}+\frac{\partial^{2} \psi}{\partial r_{2} \partial r_{12}} 0+\frac{\partial^{2}}{\partial r_{12}^{2}}\left(-\frac{x_{2}-x_{1}}{r_{12}}\right)
\end{aligned}
$$

and

$$
\begin{gathered}
\frac{\partial^{2} \psi}{\partial x_{1}^{2}=} \\
+\left(\frac{\partial^{2} \psi}{\partial r_{1}^{2}} \frac{x_{1}}{r_{1}}+\frac{\partial^{2} \psi}{\partial r_{12} \partial r_{1}}\left(\frac{1}{r_{1}}-\frac{x_{1}^{2}}{r_{1}^{3}}\right)\right. \\
\left.+\left(\frac{x_{2}-x_{1}}{r_{12}}\right)\right) \frac{x_{1}}{r_{1}}+\frac{\partial \psi}{\partial r_{12}}\left(\frac{1}{r_{12}}-\frac{\left(x_{2}-x_{1}\right)^{2}}{r_{12}^{3}}\right) \\
\left.r_{1} \partial r_{12} \frac{x_{1}}{r_{1}}+\frac{\partial^{2}}{\partial r_{12}^{2}}\left(-\frac{x_{2}-x_{1}}{r_{12}}\right)\right)\left(-\frac{x_{2}-x_{1}}{r_{12}}\right) .
\end{gathered}
$$

This produces the result for the second derivative

$$
\begin{aligned}
\frac{\partial^{2} \psi}{\partial x_{1}^{2}}=\frac{\partial^{2} \psi}{\partial r_{1}^{2}} \frac{x_{1}^{2}}{r_{1}^{2}}+ & \frac{\partial \psi}{\partial r_{1}}\left(\frac{1}{r_{1}}-\frac{x_{1}^{2}}{r_{1}^{3}}\right)+\frac{\partial^{2} \psi}{\partial r_{12}^{2}} \frac{\left(x_{2}-x_{1}\right)^{2}}{r_{12}^{2}} \\
+ & \frac{\partial \psi}{\partial r_{12}}\left(\frac{1}{r_{12}}-\frac{\left(x_{2}-x_{1}\right)^{2}}{r_{12}^{3}}\right) \\
& +2 \frac{\partial^{2} \psi}{\partial r_{1} \partial r_{12}} \frac{x_{1}\left(x_{1}-x_{2}\right)}{r_{1} r_{12}} .
\end{aligned}
$$

One needs to repeat this procedure for all second derivatives of the Laplacian $\left(\frac{\partial^{2} \psi}{\partial x_{1}^{2}}\right.$, $\left.\frac{\partial^{2} \psi}{\partial y_{1}^{2}}, \frac{\partial^{2} \psi}{\partial z_{1}^{2}}, \frac{\partial^{2} \psi}{\partial x_{2}^{2}}, \frac{\partial^{2} \psi}{\partial y_{2}^{2}}, \frac{\partial^{2} \psi}{\partial z_{2}^{2}}\right)$. One finds the others to be

$$
\begin{aligned}
\frac{\partial^{2} \psi}{\partial y_{1}^{2}}=\frac{\partial^{2} \psi}{\partial r_{1}^{2}} \frac{y_{1}^{2}}{r_{1}^{2}}+ & \frac{\partial \psi}{\partial r_{1}}\left(\frac{1}{r_{1}}-\frac{y_{1}^{2}}{r_{1}^{3}}\right)+\frac{\partial^{2} \psi}{\partial r_{12}^{2}} \frac{\left(y_{2}-y_{1}\right)^{2}}{r_{12}^{2}} \\
+ & \frac{\partial \psi}{\partial r_{12}}\left(\frac{1}{r_{12}}-\frac{\left(y_{2}-y_{1}\right)^{2}}{r_{12}^{3}}\right) \\
& +2 \frac{\partial^{2} \psi}{\partial r_{1} \partial r_{12}} \frac{y_{1}\left(y_{1}-y_{2}\right)}{r_{1} r_{12}}, \\
\frac{\partial^{2} \psi}{\partial z_{1}^{2}}=\frac{\partial^{2} \psi}{\partial r_{1}^{2}} \frac{z_{1}^{2}}{r_{1}^{2}}+ & \frac{\partial \psi}{\partial r_{1}}\left(\frac{1}{r_{1}}-\frac{z_{1}^{2}}{r_{1}^{3}}\right)+\frac{\partial^{2} \psi}{\partial r_{12}^{2}} \frac{\left(z_{2}-z_{1}\right)^{2}}{r_{12}^{2}} \\
+ & \frac{\partial \psi}{\partial r_{12}}\left(\frac{1}{r_{12}}-\frac{\left(z_{2}-z_{1}\right)^{2}}{r_{12}^{3}}\right) \\
& +2 \frac{\partial^{2} \psi}{\partial r_{1} \partial r_{12}} \frac{z_{1}\left(z_{1}-z_{2}\right)}{r_{1} r_{12}},
\end{aligned}
$$




$$
\begin{aligned}
\frac{\partial^{2} \psi}{\partial x_{2}^{2}}=\frac{\partial^{2} \psi}{\partial r_{1}^{2}} \frac{x_{2}^{2}}{r_{1}^{2}}+ & \frac{\partial \psi}{\partial r_{1}}\left(-\frac{1}{r_{1}}-\frac{x_{2}^{2}}{r_{1}^{3}}\right)+\frac{\partial^{2} \psi}{\partial r_{12}^{2}} \frac{\left(x_{2}-x_{1}\right)^{2}}{r_{12}^{2}} \\
+ & \frac{\partial \psi}{\partial r_{12}}\left(-\frac{1}{r_{12}}-\frac{\left(x_{2}-x_{1}\right)^{2}}{r_{12}^{3}}\right) \\
+ & 2 \frac{\partial^{2} \psi}{\partial r_{1} \partial r_{12}} \frac{x_{2}\left(x_{2}-x_{1}\right)}{r_{1} r_{12}} \\
\frac{\partial^{2} \psi}{\partial y_{2}^{2}}=\frac{\partial^{2} \psi}{\partial r_{1}^{2}} \frac{y_{2}^{2}}{r_{1}^{2}}+ & \frac{\partial \psi}{\partial r_{1}}\left(-\frac{1}{r_{1}}-\frac{y_{2}^{2}}{r_{1}^{3}}\right)+\frac{\partial^{2} \psi}{\partial r_{12}^{2}} \frac{\left(y_{2}-y_{1}\right)^{2}}{r_{12}^{2}} \\
+ & \frac{\partial \psi}{\partial r_{12}}\left(-\frac{1}{r_{12}}-\frac{\left(y_{2}-y_{1}\right)^{2}}{r_{12}^{3}}\right) \\
+ & 2 \frac{\partial^{2} \psi}{\partial r_{1} \partial r_{12}} \frac{y_{2}\left(y_{2}-y_{1}\right)}{r_{1} r_{12}}
\end{aligned}
$$

and

$$
\begin{aligned}
\frac{\partial^{2} \psi}{\partial z_{2}^{2}}=\frac{\partial^{2} \psi}{\partial r_{1}^{2}} \frac{z_{2}^{2}}{r_{1}^{2}}+ & \frac{\partial \psi}{\partial r_{1}}\left(-\frac{1}{r_{1}}-\frac{z_{2}^{2}}{r_{1}^{3}}\right)+\frac{\partial^{2} \psi}{\partial r_{12}^{2}} \frac{\left(z_{2}-z_{1}\right)^{2}}{r_{12}^{2}} \\
+ & \frac{\partial \psi}{\partial r_{12}}\left(-\frac{1}{r_{12}}-\frac{\left(z_{2}-z_{1}\right)^{2}}{r_{12}^{3}}\right) \\
+ & 2 \frac{\partial^{2} \psi}{\partial r_{1} \partial r_{12}} \frac{z_{2}\left(z_{2}-z_{1}\right)}{r_{1} r_{12}}
\end{aligned}
$$

Combining these together and noting that

$$
r_{1}^{2}-r_{2}^{2}+r_{12}^{2}=2\left[x_{1}\left(x_{1}-x_{2}\right)+y_{1}\left(y_{1}-y_{2}\right)+z_{1}\left(z_{1}-z_{2}\right)\right]
$$

yields

$$
\begin{gathered}
-\frac{1}{2} \nabla_{r_{1}}^{2}-\frac{1}{2} \nabla_{r_{2}}^{2}=-\frac{1}{2} \frac{\partial^{2}}{\partial r_{1}^{2}}-\frac{1}{2} \frac{\partial^{2}}{\partial r_{2}^{2}}-\frac{\partial^{2}}{\partial r_{12}^{2}}-\frac{1}{r_{1}} \frac{\partial}{\partial r_{1}}-\frac{1}{r_{2}} \frac{\partial}{\partial r_{2}}-\frac{2}{r_{12}} \frac{\partial}{\partial r_{12}} \\
-\frac{r_{1}^{2}-r_{2}^{2}+r_{12}^{2}}{2 r_{1} r_{12}} \frac{\partial^{2}}{\partial r_{1} \partial r_{12}}-\frac{r_{2}^{2}-r_{1}^{2}+r_{12}^{2}}{2 r_{2} r_{12}} \frac{\partial^{2}}{\partial r_{2} \partial r_{12}} .
\end{gathered}
$$

Thus, the Hamiltonian in $r_{1}, r_{2}, r_{12}$ basis is, 


$$
\begin{gathered}
H=-\frac{1}{2} \frac{\partial^{2}}{\partial r_{1}^{2}}-\frac{1}{2} \frac{\partial^{2}}{\partial r_{2}^{2}}-\frac{\partial^{2}}{\partial r_{12}^{2}}-\frac{1}{r_{1}} \frac{\partial}{\partial r_{1}}-\frac{1}{r_{2}} \frac{\partial}{\partial r_{2}}-\frac{2}{r_{12}} \frac{\partial}{\partial r_{12}} \\
-\frac{r_{1}^{2}-r_{2}^{2}+r_{12}^{2}}{2 r_{1} r_{12}} \frac{\partial^{2}}{\partial r_{1} \partial r_{12}}-\frac{r_{2}^{2}-r_{1}^{2}+r_{12}^{2}}{2 r_{2} r_{12}} \frac{\partial^{2}}{\partial r_{2} \partial r_{12}} \\
-\left(\frac{Z}{r_{1}}+\frac{Z}{r_{2}}-\frac{1}{r_{12}}\right)
\end{gathered}
$$

The volume element in this coordinate system is

$$
V=8 \pi^{2} \int_{0}^{\infty} d r_{1} \int_{0}^{\infty} d r_{2} \int_{\left|r_{1}-r_{2}\right|}^{r_{1}+r_{2}} d r_{12} r_{1} r_{2} r_{12}
$$

One might also like to transform into the Hylleraas $s, t, u$ basis,

$$
\begin{aligned}
s & =r_{1}+r_{2} \\
t & =r_{1}-r_{2} \\
u & =r_{12}
\end{aligned}
$$

which implies

$$
\begin{aligned}
& r_{1}=\frac{1}{2}(s+t) \\
& r_{2}=\frac{1}{2}(s-t) \\
& r_{12}=u .
\end{aligned}
$$

We can use the same chain rule procedure as before,

$$
\frac{\partial \psi}{\partial x_{1}}=\frac{\partial \psi}{\partial s} \frac{\partial s}{\partial r_{1}}+\frac{\partial \psi}{\partial t} \frac{\partial t}{\partial r_{1}}+\frac{\partial \psi}{\partial u} \frac{\partial u}{\partial r_{1}}
$$

We will leave the details to the reader. The result is 


$$
\begin{array}{cc}
H= & -\frac{\partial^{2}}{\partial s^{2}}-\frac{\partial^{2}}{\partial u^{2}}-\frac{\partial^{2}}{\partial t^{2}} \\
-\frac{4 s}{s^{2}-t^{2}} \frac{\partial}{\partial s}-\frac{4 s}{t^{2}-s^{2}} \frac{\partial}{\partial t}-\frac{2}{u} \frac{\partial}{\partial u} \\
-\frac{2 s\left(u^{2}-t^{2}\right)}{u\left(s^{2}-t^{2}\right)} \frac{\partial^{2}}{\partial s \partial u}-\frac{2 t\left(s^{2}-u^{2}\right)}{u\left(s^{2}-t^{2}\right)} \frac{\partial^{2}}{\partial t \partial u} \\
-\left(\frac{4 s Z}{s^{2}-t^{2}}-\frac{1}{u}\right)
\end{array}
$$

The volume element in this coordinate system is

$$
V=2 \pi^{2} \int_{0}^{\infty} d s \int_{0}^{s} d u \int_{0}^{u} d t u\left(s^{2}-t^{2}\right) .
$$

